\title{
The Value in Muddling Around Modelling
}

\author{
Marian Petre \\ Centre for Research in Computing, The Open University, UK \\ m.petre@open.ac.uk
}

\begin{abstract}
Software is a designed artifact. In other design disciplines, such as building architecture, there is a well-established tradition of design studies which inform not only the discipline itself but also tool design, processes, and collaborative work. This talk considers software from such a 'design studies' perspective. The talk will present a series of observations from empirical studies of expert software designers, and will draw on examples from actual professional practice. It will consider what experts' mental imagery, software visualisations, and sketches suggest about software design thinking. It will discuss which representations designers use when allowed to choose freely, how designers' informal representations relate to the formal representations from their discipline, how the character of their informal representations facilitates design discussions, and why many of the functions afforded by their sketching are not well supported by existing CAD systems. It will consider what the observations and sketches reveal about requirements for an idea-capture tool that supports collaborative design. The talk will also discuss some of the deliberate practices experts use to promote innovation. Finally, it will open discussion on the tensions between observed software design practices and received methodology in software engineering.
\end{abstract}

Keywords: empirical studies, expert design, software design, flexible modeling, software engineering practice. 